\title{
Semi-Blind Adaptive Space-Time Shift Keying Systems Based on Iterative Channel Estimation and Data Detection
}

\author{
Peichang Zhang, Indrakshi Dey, Shinya Sugiura and Sheng Chen \\ School of Electronics and Computer Science \\ University of Southampton \\ Southampton SO17 1BJ, UK \\ E-mails: \{pz3g09, id2g09, ss07r, sqc $\} @$ ecs.soton.ac.uk
}

\begin{abstract}
We develop a semi-blind adaptive space-time shift keying (STSK) based multiple-input multiple-output system using a low-complexity iterative channel estimation and data detection scheme. We first employ the minimum number of STSK training blocks, which is related to the number of transmitter antennas, to obtain a rough least square channel estimate (LSCE). Low-complexity single-stream maximum likelihood (ML) data detection is then carried out based on the initial LSCE and the detected data are utilised to refine the decision-directed LSCE. We show that a few iterations are sufficient to approach the optimal ML detection performance obtained with the aid of perfect channel state information.
\end{abstract}

\section{INTRODUCTION}

A unified multiple-input multiple-output (MIMO) architecture based on the generic space-time shift keying (STSK) concept [1], [2] has recently been proposed, which includes spatial modulation (SM) and space shift keying (SSK) [3][6], orthogonal space-time block codes [7], Bell Lab's layered space-time scheme [8] as well as linear dispersion codes [9] as its special cases. Unlike the SM and SSK schemes [3]-[6], which only utilise the spatial dimension, the STSK scheme exploits both the space and time dimensions. More specifically, the STSK system is based on the activation of the appropriately indexed space-time dispersion matrices within each STSK block duration, instead of the indexed antennas at each symbol duration, as in the SM and SSK systems of [3]-[6]. The STSK scheme is capable of striking a flexible diversity versus multiplexing gain trade off, which is achieved by optimising both the number and size of the dispersion matrices as well as the number of transmit and receive antennas. In particular, the STSK system is capable of exploiting both transmit and receive diversity gains, unlike the SM and SSK schemes, which can only attain a receive diversity gain. Moreover, like the SM system, the STSK scheme does not impose inter-antenna, i.e. interchannel interference (ICI). Consequently, the single-antenna based maximum likelihood (ML) detector of [5] can readily be employed in the STSK system to attain optimal ML detection at a low complexity. In this contribution, we study the coherent STSK (CSTSK) based MIMO system.

In general, the ability of a coherent MIMO system to approach its capacity heavily relies on the accuracy of the channel state information (CSI). Training based adaptive schemes are capable of accurately estimating a MIMO channel at the expense of considerable reduction in system throughput, since a large training overhead is required to obtain a reasonably accurate CSI estimate. Blind methods not only impose high complexity and slow convergence, but also suffer from unavoidable estimation and decision ambiguities [10]. Semi-blind methods offer attractive practical means of implementing adaptive MIMO systems. In the semi-blind methods of [11]-[14], a few training symbols are used to provide an initial MIMO channel estimate. Then the channel estimator as well as the data detector iteratively exchange their information, where the channel estimator relies on decisiondirected adaptation. In the scheme of [15], [16], aided by an initial training-based MIMO channel estimate, blind joint ML data detection and channel estimation is carried out by a computational intelligence based optimisation algorithm. In these studies, however, the MIMO systems induce ICI, and ML data detection has to be carried out, for example, with the aid of sphere-decoding based algorithms [17], [18], which may be computationally expensive. High-complexity ML data detection coupled with a large number of iterations to achieve convergence imposes considerable computational requirements in these previous semi-blind methods.

We exploit the inherent low-complexity of ML data detection in STSK systems and propose a semi-blind iterative channel estimation and data detection scheme for CSTSK systems. In order to maintain a high system throughput, the minimum number of STSK training blocks, which is determined by the number of transmitter antennas, is utilised to provide a rough least square channel estimate (LSCE). Then low-complexity single-antenna based ML data detection is carried out based on the initial LSCE, and the detected data are then re-modulated and used for the decision-directed LSCE update. Our results demonstrate that a few iterations, typically no more than five, are sufficient to approach the optimal detection performance obtained with perfect CSI. The remainder of this contribution is organised as follows. Section II describes the CSTSK system model and the lowcomplexity optimal ML data detection algorithm, while the proposed semi-blind iterative channel estimation and data detection scheme is presented in Section III. Simulation results are given in Section IV, and our conclusions are summarised in Section V.

\section{Coherent STSK System Model}

We consider the CSTSK based MIMO system [2], which employs $N_{T}$ antennas at the transmitter and $N_{R}$ antennas at the receiver for communication in a frequency-flat Rayleigh 


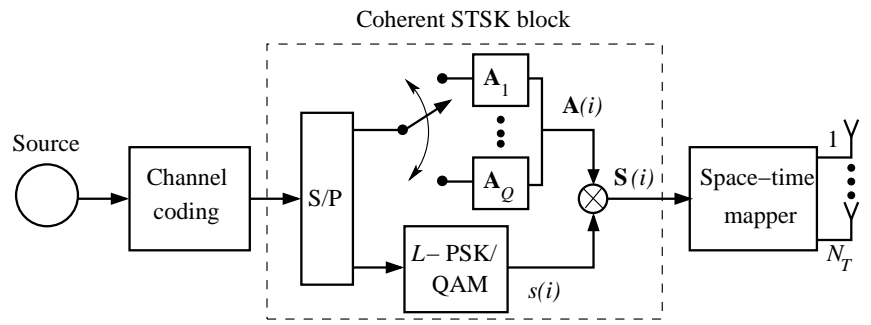

Fig. 1. Transmitter structure of the coherent STSK scheme.

fading environment. Let $\mathbb{C}$ denote the field of complex numbers, $T_{n}$ be the number of time slots, and $i$ indicate the STSK block index. Fig. 1 depicts the CSTSK system's transmitter, where the information bit sequence is first coded by a standard channel coder, and the coded bit sequence are then mapped into the STSK signal matrices $\mathbf{S}(i) \in \mathbb{C}^{N_{T} \times T_{n}}$. Specifically, each STSK block $\mathbf{S}(i)$ is given by [1], [2]

$$
\mathbf{S}(i)=s(i) \mathbf{A}(i),
$$

where

$$
s(i) \in \mathcal{S}=\left\{s_{l} \in \mathbb{C}, 1 \leq l \leq L\right\}
$$

is the complex-valued symbol of the conventional modulation scheme employed, such as $L$-PSK or $L$-QAM, which is associated with $\log _{2}(L)$ input bits, while

$$
\mathbf{A}(i) \in \mathcal{A}=\left\{\mathbf{A}_{q} \in \mathbb{C}^{N_{T} \times T_{n}}, 1 \leq q \leq Q\right\}
$$

is selected from the $Q$ pre-assigned dispersion matrices $\mathbf{A}_{q}$, $1 \leq q \leq Q$, according to $\log _{2}(Q)$ input bits. The $m$ th row's elements of $\mathbf{S}(i)$ are transmitted from the $m$ th transmitter antenna in $T_{n}$ time slots. Since a total of $\log _{2}(L \cdot Q)$ bits are mapped to each STSK block, the normalised throughput per time-slot, $R$, of this CSTSK scheme can be expressed as

$$
R=\frac{\log _{2}(Q \cdot L)}{T_{n}}[\text { bits/symbol]. }
$$

The design of dispersion matrices is an important research subject entirely in itself [19]. To maintain a unity average transmission power for each STSK block, each of the $Q$ dispersion matrices must meet the power constraint of

$$
\operatorname{tr}\left[\mathbf{A}_{q}^{H} \mathbf{A}_{q}\right]=T_{n}, 1 \leq q \leq Q,
$$

where $\operatorname{tr}[\cdot]$ denotes the matrix trace operator. Given the CSTSK parameters of $\left(N_{T}, N_{R}, T_{n}, Q\right)$ as well as the constellation size $L$, a numerical search is adopted in [2] to obtain the set of $Q$ dispersion matrices $\mathcal{A}$ by maximising the discrete-input continuous-output memoryless channel capacity [20] subject to the constraints (5).

The received signal matrix $\mathbf{Y}(i) \in \mathbb{C}^{N_{R} \times T_{n}}$ can be expressed by the following MIMO model

$$
\mathbf{Y}(i)=\mathbf{H} \mathbf{S}(i)+\mathbf{V}(i),
$$

where $\mathbf{H} \in \mathbb{C}^{N_{R} \times N_{T}}$ and $\mathbf{V}(i) \in \mathbb{C}^{N_{R} \times T_{n}}$ denote the channel matrix and the $i$ th noise matrix, respectively. We assume that each element of $\mathbf{H}$ obeys the complex-valued Gaussian distribution with zero mean and a variance of 0.5 per dimension, which is denoted as $\mathcal{C N}(0,1)$. The channel matrix $\mathbf{H}$ is assumed to remain constant for at least $T_{n}$ time slots. Each element of $\mathbf{V}(i)$ obeys the complex-valued zero-mean Gaussian distribution of $\mathcal{C N}\left(0, N_{\mathrm{o}}\right)$ with a noise variance of $N_{\mathrm{o}} / 2$ per dimension. The signal to noise ratio (SNR) of the system is defined as SNR $=E_{\mathrm{S}} / N_{\mathrm{o}}$, where $E_{\mathrm{S}}$ is the average symbol energy of the modulation scheme (2).

We introduce the following notations

$$
\begin{aligned}
\overline{\mathbf{y}}(i)=\operatorname{vec}[\mathbf{Y}(i)] & \in \mathbb{C}^{N_{R} T_{n} \times 1} \\
\overline{\mathbf{H}}=\mathbf{I}_{T_{n}} \otimes \mathbf{H} & \in \mathbb{C}^{N_{R} T_{n} \times N_{T} T_{n}} \\
\overline{\mathbf{v}}(i)=\operatorname{vec}[\mathbf{V}(i)] & \in \mathbb{C}^{N_{R} T_{n} \times 1} \\
\boldsymbol{\Theta}=\left[\operatorname{vec}\left[\mathbf{A}_{1}\right] \cdots \operatorname{vec}\left[\mathbf{A}_{Q}\right]\right] & \in \mathbb{C}^{N_{T} T_{n} \times Q}
\end{aligned}
$$

where vec [.] is the vector stacking operator, $\mathbf{I}_{M}$ the $M \times M$ identity matrix and $\otimes$ the Kronecker product. Further define the equivalent transmitted signal vector $\mathbf{k}(i) \in \mathbb{C}^{Q \times 1}$ as

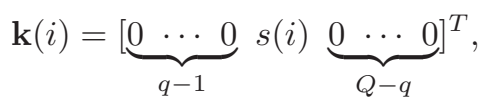

where the modulated symbol $s(i)$ is situated in the $q$ th element. The index $q$ corresponds to the index of the dispersion matrix $\mathbf{A}_{q}$ activated during the $i$ th STSK block, and the transmitted signal vector $\mathbf{k}(i)$ takes its value from the set

$$
\mathcal{K}=\left\{\mathbf{k}_{q, l} \in \mathbb{C}^{Q \times 1}, 1 \leq q \leq Q, 1 \leq l \leq L\right\},
$$

which contains the $Q \cdot L$ legitimate transmitted signal vectors,

$\mathbf{k}_{q, l}=[\underbrace{0 \cdots 0}_{q-1} s_{l} \underbrace{0 \cdots 0}_{Q-q}]^{T}, 1 \leq q \leq Q, 1 \leq l \leq L$,

where $s_{l}$ is the $l$ th symbol in the $L$-point constellation $\mathcal{S}$. With the notations (7) to (11), the signal model equivalent to (6) is

$$
\overline{\mathbf{y}}(i)=\overline{\mathbf{H}} \boldsymbol{\Theta} \mathbf{k}(i)+\overline{\mathbf{v}}(i) .
$$

When $\mathbf{H}$ is known at the receiver, data detection can be carried out very efficiently [2]. This is because the equivalent system model (14) is free from the effects of ICI, and the low-complexity single-antenna based ML detector of [5] can readily be applied to achieve optimal data detection. Let $(q, l)$ correspond to the specific input bits of a STSK block, which are mapped to the $l$ th symbol $s_{l}$ and the $q$ th dispersion matrix $\mathbf{A}_{q}$. Then the ML estimates $(\hat{q}, \hat{l})$ are given by

$$
\begin{aligned}
(\hat{q}, \hat{l}) & =\arg \min _{\substack{1 \leq q \leq Q \\
1 \leq l \leq L}}\left\|\overline{\mathbf{y}}(i)-\overline{\mathbf{H}} \boldsymbol{\Theta} \mathbf{k}_{q, l}\right\|^{2} \\
& =\arg \min _{\substack{1 \leq q \leq Q \\
1 \leq l \leq L}}\left\|\overline{\mathbf{y}}(i)-s_{l}(\overline{\mathbf{H}} \boldsymbol{\Theta})_{q}\right\|^{2},
\end{aligned}
$$

where $(\overline{\mathbf{H}} \Theta)_{q}$ denotes the $q$ th column of the matrix $\overline{\mathbf{H}} \Theta$. The detected bits are passed through the channel decoder to recover the corresponding information bits.

The calculation of $(\overline{\mathbf{H}} \Theta)_{q}$ for $1 \leq q \leq Q$ requires $Q T_{n} N_{R} 4 N_{T}$ real-valued multiplications and $Q T_{n} N_{R}\left(4 N_{T}-2\right)$ real-valued additions. In a slow-fading environment, this calculation can be reused during the channel's coherent time. The detection of a STSK block or $\log _{2}(Q \cdot L)$ bits using (15) requires $6 L Q N_{R} T_{n}$ real-valued multiplications and $L Q\left(6 N_{R} T_{n}-1\right)$ real-valued additions. Let the channel's coherence time be the duration of $\tau$ STSK blocks. Then the total complexity of detecting $\tau \log _{2}(Q \cdot L)$ bits is detailed in Table I and summarised as follows

$$
C_{\mathrm{ML}} \approx 4 Q T_{n} N_{R}\left(3 \tau L+2 N_{T}\right) \text { [Flops]. }
$$


TABLE I

COMPLEXITY OF DETECTING $\tau$ STSK BLOCKS, ASSUMING THAT THE CHANNEL'S COHERENT TIME LASTS THE DURATION OF $\tau$ STSK BLOCKS.

\begin{tabular}{l|r|r}
\hline Operation & Real-valued multiplications & Real-valued additions \\
\hline calculating $(\overline{\mathbf{H}} \Theta)_{q}, 1 \leq q \leq Q$ & $4 Q T_{n} N_{R} N_{T}$ & $4 Q T_{n} N_{R} N_{T}-2 Q T_{n} N_{R}$ \\
detecting one STSK block & $6 L Q T_{n} N_{R}$ & $6 L Q T_{n} N_{R}-L Q$ \\
total complexity of detecting $\tau \log _{2}(Q \cdot L)$ bits & $Q T_{n} N_{R}\left(6 \tau L+4 N_{T}\right)$ & $Q T_{n} N_{R}\left(6 \tau L+4 N_{T}-2\right)-\tau L Q$ \\
\hline
\end{tabular}

\section{Semi-Blind Iterative Channel Estimation And DATA DETECTION}

Assume that the number of available training blocks is $M$ and the training data are arranged as

$$
\begin{aligned}
\mathbf{Y}_{\mathrm{t} M} & =\left[\begin{array}{llll}
\mathbf{Y}(1) & \mathbf{Y}(2) & \cdots & \mathbf{Y}(M)
\end{array}\right] \\
\mathbf{S}_{\mathrm{t} M} & =\left[\begin{array}{llll}
\mathbf{S}(1) & \mathbf{S}(2) & \cdots & \mathbf{S}(M)
\end{array}\right]
\end{aligned}
$$

Then the LSCE based on $\left(\mathbf{Y}_{\mathrm{t} M}, \mathbf{S}_{\mathrm{t} M}\right)$ is given by

$$
\hat{\mathbf{H}}_{\mathrm{LSCE}}=\mathbf{Y}_{\mathrm{t} M} \mathbf{S}_{\mathrm{t} M}^{H}\left(\mathbf{S}_{\mathrm{t} M} \mathbf{S}_{\mathrm{t} M}^{H}\right)^{-1} \text {. }
$$

To maintain a high system throughput, we should only use the minimum number of STSK training blocks. In order for $\mathbf{S}_{\mathrm{t} M} \mathbf{S}_{\mathrm{t} M}^{H}$ to have the full rank of $N_{T}$, it is necessary that $M \cdot T_{n} \geq N_{T}$ and this requires a minimum of

$$
M=\left\lceil\frac{N_{T}}{T_{n}}\right\rceil
$$

training blocks, where $\lceil x\rceil$ denotes the integer ceiling operation that is larger than or equal to $x$. Thus we will choose the number of initial training blocks according to (20). For example, if $N_{T}=4$ and $T_{n}=2$, then the minimum number of STSK training blocks is $M=2$. Given such a small training data set, the accuracy of the LSCE (19) will be poor and the achievable bit error ratio (BER) of the ML detector (15) based on this rough CSI estimate will also be poor. We propose to use the following iterative channel estimation and data detection scheme to improve the detection and estimation performance. Let the observation data for the ML detector be denoted as

$$
\mathbf{Y}_{\mathrm{d} \tau}=\left[\begin{array}{llll}
\mathbf{Y}(1) & \mathbf{Y}(2) & \cdots & \mathbf{Y}(\tau)
\end{array}\right]
$$

and fix the number of iterations to $I_{\max }$.

\section{Semi-Blind Iterative Algorithm}

1) Set the iteration index $t=0$ and the channel estimate $\tilde{\mathbf{H}}^{(t)}=\hat{\mathbf{H}}_{\mathrm{LSCE}}$;

2) Given $\tilde{\mathbf{H}}^{(t)}$, perform $\mathrm{ML}$ data detection on $\mathbf{Y}_{\mathrm{d} \tau}$ and carry out the channel decoding on the detected bits. The corresponding detected information bits, after passing through the channel coder again, are remodulated to yield

$$
\hat{\mathbf{S}}_{\mathrm{e} \tau}^{(t)}=\left[\begin{array}{llll}
\hat{\mathbf{S}}^{(t)}(1) & \hat{\mathbf{S}}^{(t)}(2) & \cdots & \hat{\mathbf{S}}^{(t)}(\tau)
\end{array}\right] ;
$$

3) Update the channel estimate with

$$
\tilde{\mathbf{H}}^{(t+1)}=\mathbf{Y}_{\mathrm{d} \tau}\left(\hat{\mathbf{S}}_{\mathrm{e} \tau}^{(t)}\right)^{H}\left(\hat{\mathbf{S}}_{\mathrm{e} \tau}^{(t)}\left(\hat{\mathbf{S}}_{\mathrm{e} \tau}^{(t)}\right)^{H}\right)^{-1} ;
$$

4) Set $t=t+1$ : If $t<I_{\max }$, go to Step 2); otherwise, stop.

The total complexity of this semi-blind iterative channel estimation and data detection process is proportional to $I_{\max } \cdot\left(C_{\mathrm{ML}}+C_{\mathrm{CD}}\right)$, with $C_{\mathrm{CD}}$ being the complexity of the channel decoder. Our empirical results show that a small number of iterations is sufficient for the iterative procedure to converge, and typically $I_{\max } \leq 5$. For medium to high SNR values this iterative procedure is capable of converging to the optimal ML detection performance obtained under perfect CSI. In fact, if the initial channel estimate $\tilde{\mathbf{H}}^{(0)}$ can yield a BER below 0.1, the decision-directed channel estimator of Step 3) is capable of improving the accuracy of the channel estimate. This in turn significantly enhances the BER of the ML data detection in Step 2). Therefore, a few iterations are sufficient to attain the optimal ML solution. For low SNR values, however, some degradation from the optimal ML performance may be expected, particularly when the initial BER is higher than 0.1 . In such a situation, increasing the minimum training blocks of (20) by just one block will often ensure the convergence to the ML solution.

\section{Simulation Study}

The achievable performance was assessed using three metrics: the estimated mean square error (MSE) defined by

$$
J_{\mathrm{MSE}}(\tilde{\mathbf{H}})=\frac{1}{\tau \cdot N_{R} \cdot T_{n}} \sum_{i=1}^{\tau}\|\mathbf{Y}(i)-\tilde{\mathbf{H}} \hat{\mathbf{S}}(i)\|^{2},
$$

the mean channel estimation error (MCE) given by

$$
J_{\mathrm{MCE}}(\tilde{\mathbf{H}})=\frac{1}{N_{R} \cdot N_{T}}\|\mathbf{H}-\tilde{\mathbf{H}}\|^{2},
$$

and the achievable BER, where $\tilde{\mathbf{H}}$ is the channel estimate, $\hat{\mathbf{S}}(i)$ are the ML-detected and re-modulated data, and $\mathbf{H}$ denotes the true MIMO channel matrix. All the results were averaged over 100 channel realisations. A systematic

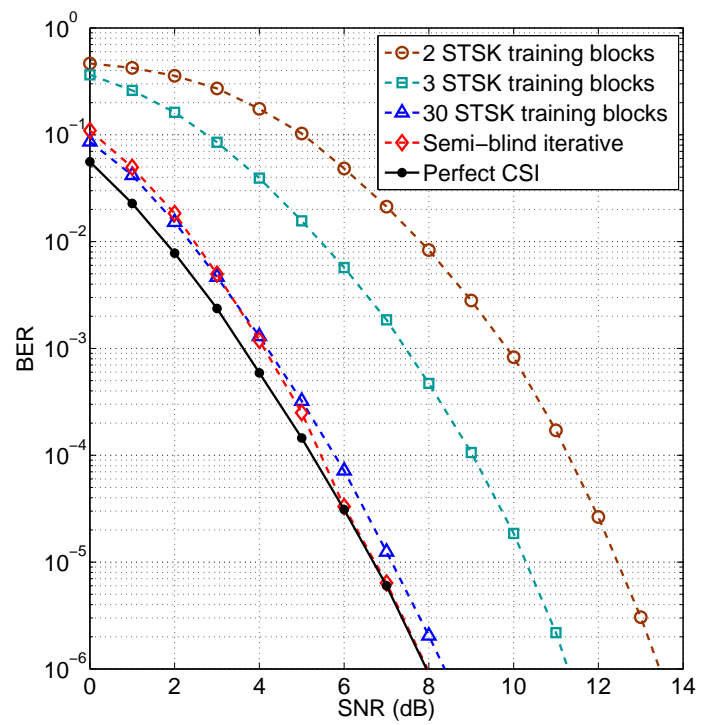

Fig. 2. BER of the proposed semi-blind scheme with 2 initial training STSK blocks, in comparison with the training-based cases using $M=2$, 3 and 30 training STSK blocks as well as the case of perfect channel state information, for the $(4,4,2,4)$ system with QPSK modulation. 
convolution code with the code rate $2 / 3$ and was used. Decoding was carried out by the hard-input hard-out Viterbi algorithm. The length of STSK data blocks for performing ML detection was $\tau=200$.

Example One. We considered a CSTSK scheme having the parameters of $\left(N_{T}=4, N_{R}=4, T_{n}=2, Q=4\right)$ and the QPSK of constellation size $L=4$. The achievable BER performance associated with assuming perfect CSI is given in Fig. 2 as the benchmark. The training-based ML detection performance using $M=2,3$ and 30, respectively, are also shown in Fig. 2 for comparison. It can be seen that the LSCEs obtained using only $M=2$ and 3 STSK training blocks were inadequate and, to approximate the true ML detection performance, more than 30 training STSK blocks were required. The performance of the proposed semi-blind scheme using $M=2$ initial training STSK blocks was then investigated. Figs. 3 and 4 characterise the convergence performance of the semi-blind iterative scheme in terms of the estimated MSE and MCE, respectively, for three different SNR values. The results shown in Figs. 3 and 4 indicate that reliable convergence was achieved in no more than five iterations. Furthermore, it can be seen from Fig. 2 that the estimated MSE converged to the noise floor $N_{\mathrm{o}}$.

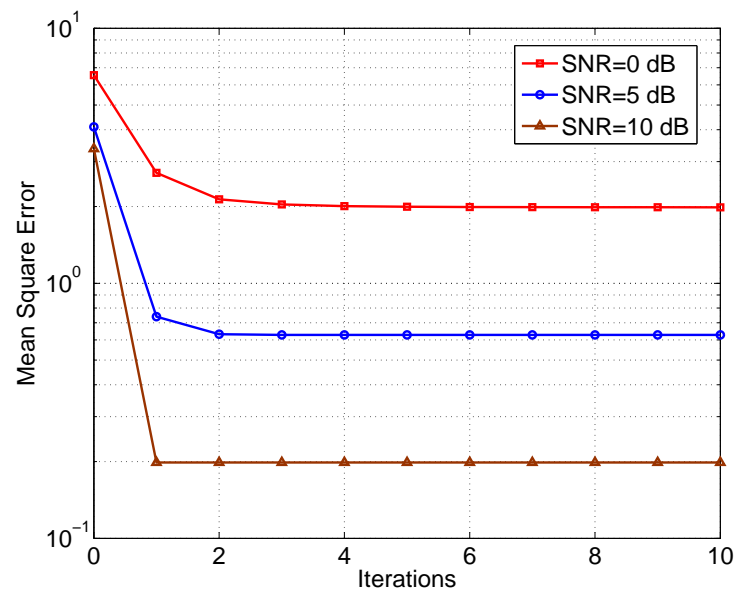

Fig. 3. Convergence of the estimated MSE for the proposed semi-blind scheme with 2 initial training STSK blocks, given different values of $E_{\mathrm{S}} / N_{\mathrm{o}}$, for the $(4,4,2,4)$ system with QPSK modulation.

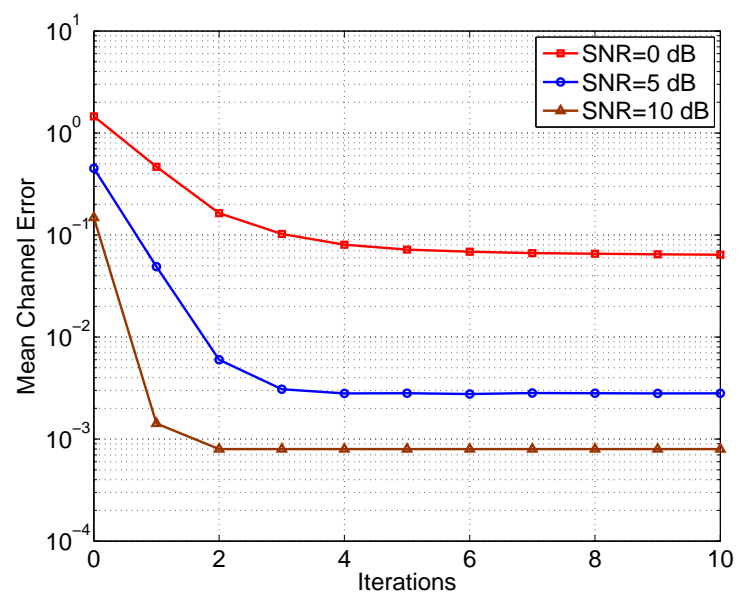

Fig. 4. Convergence of the MCE for the proposed semi-blind scheme with 2 initial training STSK blocks, given different values of $E_{\mathrm{s}} / N_{\mathrm{o}}$, for the $(4,4,2,4)$ system with QPSK modulation.

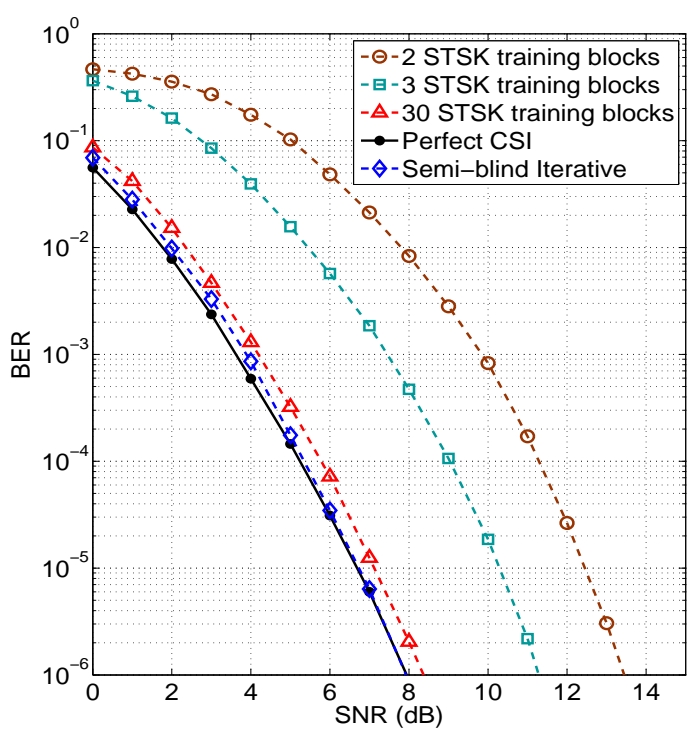

Fig. 5. BER of the proposed semi-blind scheme with 3 initial training STSK blocks, in comparison with the training-based cases using $M=2$, 3 and 30 training STSK blocks as well as the case of perfect channel state information, for the $(4,4,2,4)$ system with QPSK modulation.

The BERs of the semi-blind iterative scheme are also shown in Fig. 2. For this MIMO system, there were $N_{R}$. $N_{T}=16$ complex-valued channel taps. Two training STSK blocks corresponded to 8 training bits, and this represented a training overhead of 0.5 bit per channel. The semi-blind iterative scheme under such a low training overhead was able to approach the optimal ML performance for SNR values higher than $5 \mathrm{~dB}$, as can be seen clearly in Fig. 2. For SNR $\leq 5 \mathrm{~dB}$, some degradation from the optimal BER performance was observed. This was not surprising, since the BER achieved by the rough initial LSCE was higher than 0.1 for SNR $\leq 5 \mathrm{~dB}$. Having better initial LSCE should be able to improve the performance. We also employed $M=3$ initial STSK training blocks for the iterative semi-blind scheme,

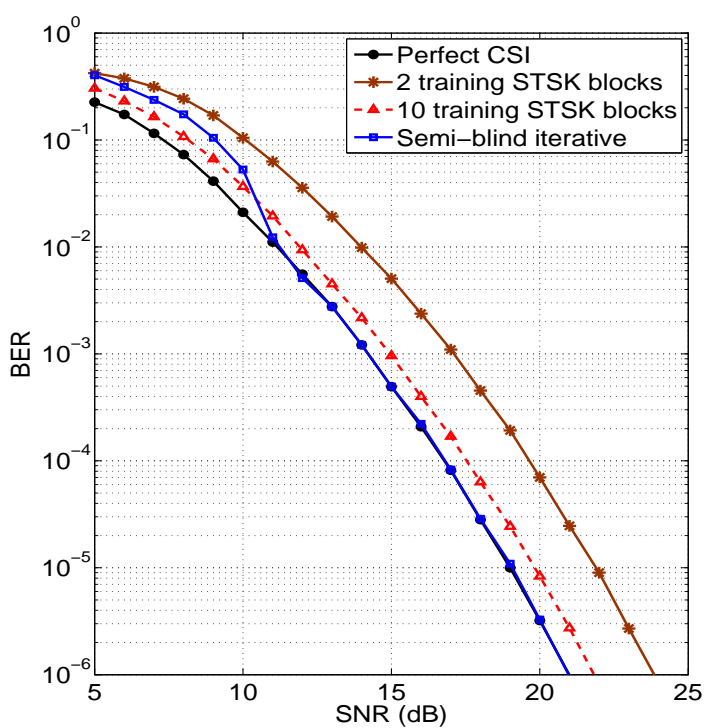

Fig. 6. BER of the proposed semi-blind scheme with 2 initial training STSK blocks, in comparison with the training-based cases using $M=2$ and 10 training STSK blocks as well as the case of perfect channel state information, for the $(4,2,2,4)$ system with 16QAM modulation. 
which still represented a very low training overhead of less than 1 bit per channel. The results obtained are shown in Fig. 5, where it can be seen that the BER performance of the semi-blind scheme now closely approximated the optimal BER performance even at low SNR values.

Example Two. We next considered a CSTSK system having the parameters of $\left(N_{T}=4, N_{R}=2, T_{n}=2, Q=4\right)$ and the 16QAM of constellation size $L=16$. Fig. 6 depicts the BERs obtained by the training-based ML detector using $M=2$ and 10, respectively, as well as the BERs of the semiblind iterative scheme with 2 initial training STSK blocks, using the performance of the ML detector associated with the perfect CSI as the benchmark. The convergence performance of the semi-blind iterative scheme with 2 initial training STSK blocks, in terms of the estimated MSE and MCE, are plotted in Figs. 7 and 8, respectively, for three different SNR values.

\section{Conclusions}

A semi-blind iterative scheme of joint channel estimation and data detection has been proposed for STSK based MIMO systems. The proposed scheme is semi-blind as it utilises the minimum number of training STSK blocks to provide a

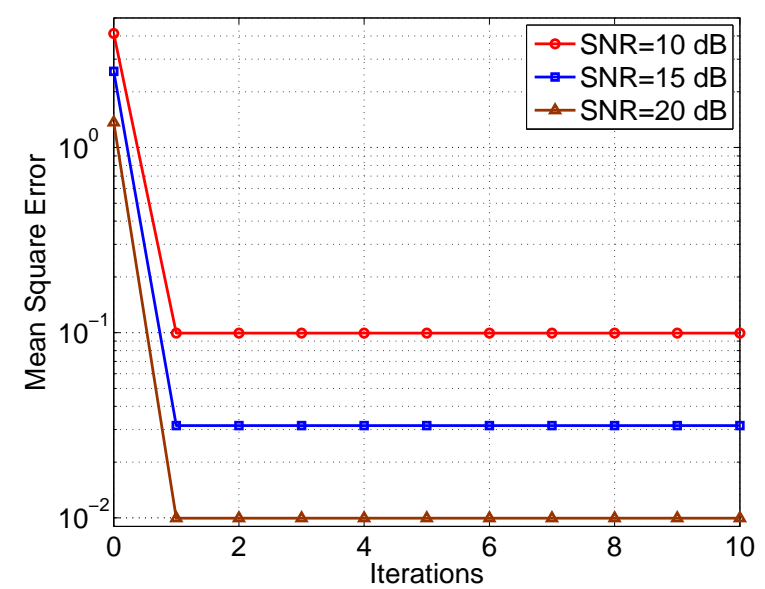

Fig. 7. Convergence of the estimated MSE for the proposed semi-blind scheme with 2 initial training STSK blocks, given different values of $E_{\mathrm{S}} / N_{\mathrm{O}}$, for the $(4,2,2,4)$ system with 16QAM modulation.

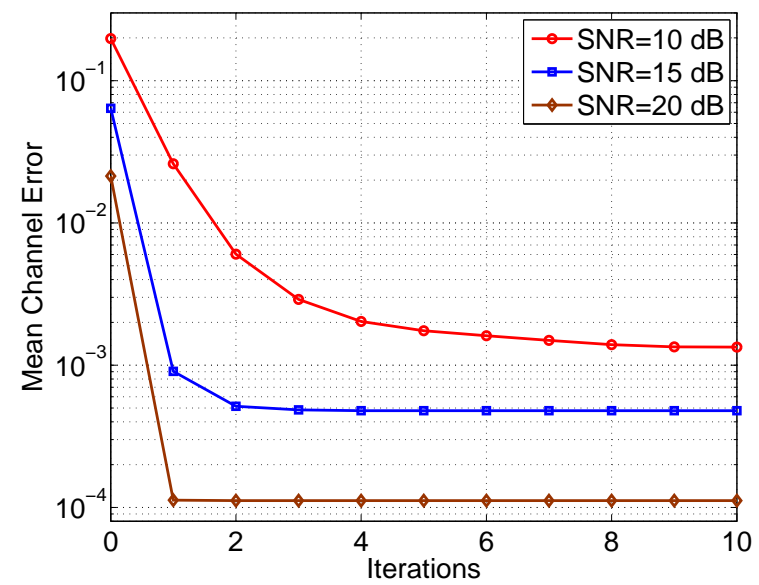

Fig. 8. Convergence of the MCE for the proposed semi-blind scheme with 2 initial training STSK blocks, given different values of $E_{\mathrm{S}} / N_{\mathrm{O}}$, for the $(4,2,2,4)$ system with 16QAM modulation. rough initial LSCE for aiding the joint iterative procedure. The proposed semi-blind joint channel estimation and ML data detection scheme is inherently low-complexity. It has been shown that the iterative procedure converges quickly, typically no more than five iterations, to the optimal ML data detection performance obtained under the perfect CSI.

\section{REFERENCES}

[1] S. Sugiura, S. Chen and L. Hanzo, "A unified MIMO architecture subsuming space shift keying, OSTBC, BLAST and LDC," in Proc. VTC 2010-Fall (Ottawa, Canada), Sept. 6-9, 2010, 5 pages.

[2] S. Sugiura, S. Chen and L. Hanzo, "Space-time shift keying: A unified MIMO architecture," in Proc. Globecom 2010 (Miami, Florida, USA), Dec.6-10, 2010, 5 pages.

[3] Y.A. Chau and S.-H. Yu, "Space modulation on wireless fading channels," in Proc. VTC-2001 Fall, Vol.3, 2001, pp.1668-1671.

[4] R.Y. Mesleh, H. Haas, S. Sinanović, C.W. Ahn and S. Yun, "Spatial modulation," IEEE Trans. Vehicular Technology, vol.57, no.4, pp.2228-2241, July 2008.

[5] J. Jeganathan, A. Ghrayeb and L. Szczecinski, "Spatial modulation: optimal detection and performance analysis," IEEE Communications Letters, vol.12, no.8, pp.545-547, August 2008.

[6] J. Jeganathan, A. Ghrayeb, L. Szczecinski and A. Ceron, "Space shift keying modulation for MIMO channels," IEEE Trans. Wireless Communications, vol.8, no.7, pp.3692-3703, July 2009.

[7] V. Tarokh, N. Seshadri and A.R. Calderbank, "Space-time codes for high data rate wireless communication: performance criterion and code construction," IEEE Trans. Information Theory, vol.44, no.2, pp.744765, March 1998.

[8] P.W. Wolniansky, G.J. Foschini, G.D. Golden and R.A. Valenzuela, "V-BLAST: An architecture for realizing very high data rates over the rich-scattering wireless channel," in Proc. Int. Sympos. Signals, Systems, and Electronics (Pisa, Italy), Sept.29-Oct.2, 1998, pp.295300 .

[9] B. Hassibi and B.M. Hochwald, "High-rate codes that are linear in space and time," IEEE Trans. Information Theory, vol.48, no.7, pp.1804-1824, July 2002.

[10] L. Tang, R.W. Liu, V.C. Soon and Y.F. Huang, "Indeterminacy and identifiability of blind identification," IEEE Trans. Circuits and Systems, vol.38, no.5, pp.499-509, May 1991.

[11] A. Medles and D.T.M. Slock, "Semiblind channel estimation for MIMO spatial multiplexing systems," in Proc. VTC2001-Fall (Atlantic City, USA), Oct.7-11, 2001, Vol.2, pp.1240-1244.

[12] C. Cozzo and B.L. Hughes, "Joint channel estimation and data detection in space-time communications," IEEE Trans. Communications, vol.51, no.8, pp.1266-1270, August 2003.

[13] S. Buzzi, M. Lops and S. Sardellitti, "Performance of iterative data detection and channel estimation for single-antenna and multipleantennas wireless communications," IEEE Trans. Vehicular Technology, vol.53, no.4, pp.1085-1104, July 2004.

[14] T. Wo, P.A. Hoeher, A. Scherb and K.D. Kammeyer, "Performance analysis of maximum-likelihood semiblind estimation of MIMO channels," in Proc. VTC2006-Spring (Melbourne, Australia), May 7-10, 2006, Vol.4, pp.1738-1742.

[15] M. Abuthinien, S. Chen and L. Hanzo, "Semi-blind joint maximum likelihood channel estimation and data detection for MIMO systems," IEEE Signal Processing Letters, vol.15, pp.202-205, 2008.

[16] H.R. Palally, S. Chen, W. Yao and L. Hanzo, "Particle swarm optimisation aided semi-blind joint maximum likelihood channel estimation and data detection for MIMO systems," in: Proc. SSPO9 (Cardiff, UK), Aug.31-Sept.3, 2009, pp.309-312.

[17] D. Pham, K.R. Pattipati, P.K. Willet and J. Luo, "An improved complex sphere decoder for V-BLAST systems," IEEE Signal Processing Letters, vol.11, no.9, pp.748-751, September 2004.

[18] J. Akhtman, A. Wolfgang, S. Chen and L. Hanzo, "An optimizedhierarchy-aided approximate Log-MAP detector for MIMO systems," IEEE Trans. Wireless Communications, vol.6, no.5, pp.1900-1909, May 2007.

[19] M. Jiang and L. Hanzo, "Unitary linear dispersion code design and optimisation for MIMO communication systems," IEEE Signal Processing Letters, vol.17, no.5, pp.497-500, May 2010.

[20] S. Ng and L. Hanzo, "On the MIMO channel capacity of multidimensional signal sets," IEEE Trans. Vehicular Technology, vol.55, no.2, pp.528-536, March 2006. 